\title{
$\omega$-3 oil intake during weight loss in obese women results in remodelling of plasma triglyceride and fatty acids
}

\author{
Gregor McCombie · Lucy M. Browning • \\ Christopher M. Titman · Molly Song • \\ John Shockcor · Susan A. Jebb · Julian L. Griffin
}

Received: 17 November 2008/Accepted: 2 April 2009/Published online: 26 April 2009

(C) Springer Science+Business Media, LLC 2009

\begin{abstract}
Previous studies have shown that a combination of weight loss and fish oil supplementation reduce cardiovascular disease and diabetes risks by increasing adiponectin and reducing triacylglyceride concentrations, while weight loss alone significantly improves insulin sensitivity and reduces inflammation. Here, a metabolomic approach, using a combination of ${ }^{1} \mathrm{H}-\mathrm{Nuclear}$ Magnetic Resonance spectroscopy, and gas and liquid chromatography and mass spectrometry, was employed to elucidate the metabolic changes in blood plasma following weight loss and fish oil supplementation. The intervention study was conducted over 24 weeks, with 93 female subjects randomised to one of three groups. Two groups followed a 12-week weight loss program, followed by a 12-week weight maintenance period and were randomised to fish or placebo oil capsules; a control group did not follow the weight loss program and were given placebo oil capsules. Lipid profiles changed dramatically upon fish oil intake and subtly across the two weight loss groups. While the fish oil supplementation increased the proportion of various phospholipid species, previously reported reductions in total triacylglycerides
\end{abstract}

Electronic supplementary material The online version of this article (doi:10.1007/s11306-009-0161-7) contains supplementary material, which is available to authorized users.

G. McCombie - C. M. Titman · M. Song · J. L. Griffin ( $\square)$ Department of Biochemistry, University of Cambridge,

Tennis Court Road, Cambridge CB2 1QW, UK

e-mail: jlg40@mole.bio.cam.ac.uk

L. M. Browning $\cdot$ S. A. Jebb

MRC Human Nutrition Research, Elsie Widdowson Laboratory,

Fulbourn Road, Cambridge CB1 9NL, UK

J. Shockcor

Waters Corporation, Milford, MA, USA
(TAGs) upon fish oil intake were shown to be driven by a reduction in a specific subset of the measured TAGs. This remodelling of triglycerides may represent further beneficial effects of fish oil supplementation.

Keywords Metabolomics - Metabonomics weight loss . Omega-3 polyunsaturated fatty acids - PUFA - NMR . LC-MS · GC-MS · Triacylglycerol

\section{Introduction}

Obesity is one of the major epidemics in western societies. In the USA obesity has doubled in adults and overweight prevalence tripled in children and adolescents from 1980 to 2002 (Ogden et al. 2006). Obesity is correlated with a higher risk of premature death (Manson et al. 1995), and in particular increased risk in the development of diabetes (Chan et al. 1994; Colditz et al. 1990), hypertension (Stamler et al. 1978) and cardiovascular disease (CVD) (Manson et al. 1990). Weight loss has proven to significantly improve insulin sensitivity, reduce blood pressure, and lower the risks associated with CVD risk and type 2 diabetes. In two independent large scale intervention programs, a weight loss of $6 \%$ as part of a lifestyle modification programme reduced the risk of progression from impaired glucose tolerance to type 2 diabetes by almost 60\% (Knowler et al. 2002; Tuomilehto et al. 2001).

Long-chain n-3 polyunsaturated fatty acids (LC n-3 PUFA), either as a supplement or when consumed as oily fish, have many beneficial effects on various measures associated with obesity and pathologies associated with the metabolic syndrome. It has been well established that fish oil intake lowers the total triacylglycerol concentration considerably more than weight loss does (Harris 1996). 
Furthermore, it has been suggested that dietary fish oil supplements protect against weight gain in animals (Kriketos et al. 2001). Fish oil has an anti-inflammatory effect, as the LC n-3 PUFA have a lower inflammatory response than the equivalent LC n-6 PUFA, even though both n-3 and n- 6 are precursors for inflammatory eicosanoids (James et al. 2000), providing an additional protective effect against CVD and diabetes, independent of BMI (Browning et al. 2004; Hill et al. 2007).

The aim of this study was to examine synergistic effects on CVD risk factors when combining weight loss with fish oil intake. We have already reported that in this study the weight loss intervention improved insulin sensitivity, reduced plasma triacylglycerides and overall inflammation, with additional decreases in blood plasma triglycerides and increased adiponectin associated with fish oil supplementation (Krebs et al. 2006). In the present analysis we have used a combined metabolomic approach to further characterise the metabolic changes that accompany combined weight loss and fish oil supplementation to better understand how these interventions produce a beneficial effect in obese individuals. Metabolomics is the study of all small molecule metabolites in a cell, tissue, organ or organism and the changes thereof due to genetic modification, disease or environmental stimuli (Weckwerth 2003; Griffin 2004). Analysis of the changing metabolite concentrations can then show which biological processes are involved following a given biological stimulus. Many of the mechanisms by which health benefits arise from weight loss or fish oil intake are yet unclear on a metabolic level. In this study we used ${ }^{1} \mathrm{H}$ Nuclear Magnetic Resonance (NMR) spectroscopy, gas and liquid chromatography and mass spectrometry (GC-MS and LC-MS) to investigate the changes in metabolism upon fish oil intake and weight loss.

\section{Materials and methods}

\subsection{Study design}

The study was approved by the local research ethics committee (Cambridgeshire, UK), and all subjects gave written informed consent. A more detailed description of intervention and study design has been previously published (Krebs et al. 2006). In brief, 93 obese women completed the 24 week study. These women (average age 44.7 years; SD 13.2; range 21-69), recruited from the community, were clinically overweight or obese (BMI $>27 \mathrm{~kg} \mathrm{~m}^{-2}$ ) and hyperinsulinaemic. They were randomised to one of three groups. One group acted as a control $(\mathrm{C})$ and were given only a placebo oil supplement, containing $2.8 \mathrm{~g}$ linoleic acid and $1.4 \mathrm{~g}$ oleic acid (provided as five $1 \mathrm{~g}$ oil capsules per day), with no weight loss program $(n=26)$. The remaining women were randomised to a 12 -week weight loss programme, designed to achieve a $10 \%$ reduction in body weight. This was followed by a 12 -week weight maintenance phase. Women randomised to the weight loss program were further randomised to receive either fish oil (FO) or placebo oil (PO) capsules. Women randomised to fish oil received five $1 \mathrm{~g}$ capsules per day $(n=35)$ containing predominantly LC n-3 PUFAs (1.3 g eicosapentaenoic acid (EPA) and $2.9 \mathrm{~g}$ docosahexaenoic acid (DHA)). The second group were given the same capsule supplement as the control group. Fasting plasma samples were taken at 3 time points: immediately before the study started (baseline, B), after 12 weeks (intermediate, I) and at the end of the study after 24 weeks (final, F) (Fig. 1). A summary of a selection clinical data as published earlier (Krebs et al. 2006) is given in Table 1.

\section{$2.2{ }^{1}$ H-NMR spectroscopy of blood plasma}

Plasma samples $(150 \mu \mathrm{l})$ were diluted with $450 \mu \mathrm{l}$ of $0.9 \%$ $\mathrm{NaCl}$ in ${ }^{2} \mathrm{H}_{2} \mathrm{O}(\mathrm{w} / \mathrm{v})$. Spectra were acquired using a $5 \mathrm{~mm}$ inverse-detection TXI probe in an 11.7 Tesla magnet (500.3 MHz proton frequency) interfaced to a Bruker AVANCE II+ spectrometer (Bruker, Coventry, UK), allowing the simultaneous measurement of $\sim 30$ metabolites which can be identified by comparison with reference spectra. Spectra were acquired at $30^{\circ} \mathrm{C}$ and were the sum of 64 transients acquired into $32 \mathrm{~K}$ data points using the 1-D version of the noesypr1d pulse sequence: Relaxation Delay (RD) $-90^{\circ}-t_{1}-90^{\circ}-t_{\mathrm{m}}-90^{\circ}$-acquire; $\mathrm{RD}=1.5 \mathrm{~s}, t_{1}=4 \mu \mathrm{s}$; $\mathrm{TR}=2 \mathrm{~s}$. The sweep width was $8,000 \mathrm{~Hz}$ and the water resonance was suppressed by low power irradiation during the mixing time $\left(t_{\mathrm{m}}\right)$ of $50 \mathrm{~ms}$ and the relaxation delay.

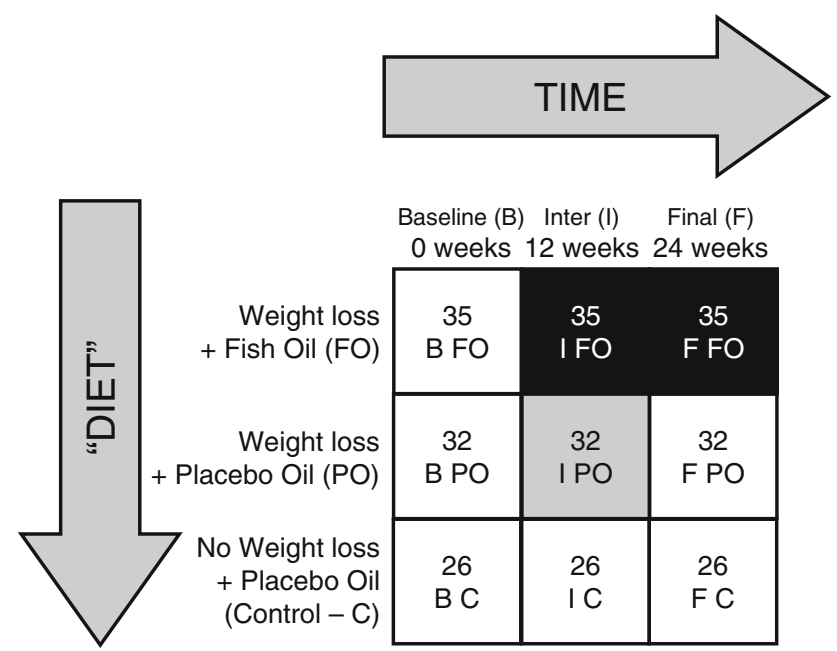

Fig. 1 Summary of the study design. Numbers in the boxes indicate the number of subjects. Black boxes indicate groups taking fish oil supplements and display the biggest differences to the other groups. The grey box shows some differences to all others due to weight loss 
Table 1 Summary of selected clinical data (SD) for the subject groups studied. For more information see (Krebs et al. 2006)

\begin{tabular}{|c|c|c|c|c|c|c|c|c|c|}
\hline & $\begin{array}{l}\text { BFO } \\
n=35\end{array}$ & $\begin{array}{l}\text { IFO } \\
n=35\end{array}$ & $\begin{array}{l}\text { FFO } \\
n=35\end{array}$ & $\begin{array}{l}\mathrm{BPO} \\
n=32\end{array}$ & $\begin{array}{l}\text { IPO } \\
n=32\end{array}$ & $\begin{array}{l}\text { FPO } \\
n=32\end{array}$ & $\begin{array}{l}\mathrm{BC} \\
n=26\end{array}$ & $\begin{array}{l}\text { IC } \\
n=26\end{array}$ & $\begin{array}{l}\text { FC } \\
n=26\end{array}$ \\
\hline Weight (Kg) & $\begin{array}{l}92.5 \\
(15.0)\end{array}$ & $\begin{array}{l}82.6 \\
(14.3)\end{array}$ & $\begin{array}{l}82.3 \\
(14.6)\end{array}$ & $\begin{array}{l}90.8 \\
(15.0)\end{array}$ & $\begin{array}{l}79.9 \\
(14.6)\end{array}$ & $\begin{array}{l}79.5 \\
(15.1)\end{array}$ & $\begin{array}{l}90.9 \\
(16.5)\end{array}$ & $\begin{array}{l}90.6 \\
(17.0)\end{array}$ & $\begin{array}{l}91.2 \\
(16.9)\end{array}$ \\
\hline Waist circumfence $(\mathrm{cm})$ & $\begin{array}{l}98.7 \\
(11.1)\end{array}$ & $\begin{array}{l}91.3 \\
(11.9)\end{array}$ & $\begin{array}{l}91.1 \\
(11.3)\end{array}$ & $\begin{array}{l}99.2 \\
(11.6)\end{array}$ & $\begin{array}{l}90.7 \\
(13.3)\end{array}$ & $\begin{array}{l}90.9 \\
(13.1)\end{array}$ & $\begin{array}{l}98.3 \\
(10.8)\end{array}$ & $\begin{array}{l}98.7 \\
(11.2)\end{array}$ & $\begin{array}{l}99.3 \\
(13.8)\end{array}$ \\
\hline $\mathrm{BMI}\left(\mathrm{Kg} \mathrm{m}^{-2}\right)$ & $\begin{array}{l}35.3 \\
(5.6)\end{array}$ & $\begin{array}{l}31.5 \\
(5.2)\end{array}$ & $\begin{array}{l}31.4 \\
(5.4)\end{array}$ & $\begin{array}{l}34.6 \\
(5.3)\end{array}$ & $\begin{array}{l}30.5 \\
(5.3)\end{array}$ & $\begin{array}{l}30.3 \\
(5.6)\end{array}$ & $\begin{array}{l}33.6 \\
(5.7)\end{array}$ & $\begin{array}{l}33.5 \\
(5.8)\end{array}$ & $\begin{array}{l}33.6 \\
(5.9)\end{array}$ \\
\hline AUC insulin (pmol/120 min) & $\begin{array}{l}47333 \\
(29191)\end{array}$ & & $\begin{array}{l}32909 \\
(18858)\end{array}$ & $\begin{array}{l}51534 \\
(35657)\end{array}$ & & $\begin{array}{l}36390 \\
(30071)\end{array}$ & $\begin{array}{l}46396 \\
(28390)\end{array}$ & & $\begin{array}{l}53460 \\
(26538)\end{array}$ \\
\hline AUC glucose $(\mathrm{mmol} / 120 \mathrm{~min})$ & $\begin{array}{l}932 \\
(212)\end{array}$ & & $\begin{array}{l}835 \\
(126)\end{array}$ & $\begin{array}{l}935 \\
(246)\end{array}$ & & $\begin{array}{l}847 \\
(243)\end{array}$ & $\begin{array}{l}872 \\
(190)\end{array}$ & & $\begin{array}{l}923 \\
(236)\end{array}$ \\
\hline Triglycerides (mmol/l) & $\begin{array}{l}1.42 \\
(0.79)\end{array}$ & & $\begin{array}{l}0.96 \\
(0.49)\end{array}$ & $\begin{array}{l}1.73 \\
(0.71)\end{array}$ & & $\begin{array}{l}1.36 \\
(0.60)\end{array}$ & $\begin{array}{l}1.48 \\
(0.99)\end{array}$ & & $\begin{array}{l}1.42 \\
(0.88)\end{array}$ \\
\hline Leptin & $\begin{array}{l}26.7 \\
(14.9)\end{array}$ & & $\begin{array}{l}16.8 \\
(10.3)\end{array}$ & $\begin{array}{l}25.9 \\
(16.8)\end{array}$ & & $\begin{array}{l}13.6 \\
(11.5)\end{array}$ & $\begin{array}{l}29.1 \\
(13.8)\end{array}$ & & $\begin{array}{l}26.0 \\
(14.5)\end{array}$ \\
\hline Adiponectin $(\mu \mathrm{g} / \mathrm{ml})$ & $\begin{array}{l}10.52 \\
(5.09)\end{array}$ & & $\begin{array}{l}12.85 \\
(6.30)\end{array}$ & $\begin{array}{l}11.43 \\
(7.59)\end{array}$ & & $\begin{array}{l}11.21 \\
(6.02)\end{array}$ & $\begin{array}{l}9.18 \\
(6.87)\end{array}$ & & $\begin{array}{l}9.71 \\
(6.75)\end{array}$ \\
\hline
\end{tabular}

Spectra were Fourier transformed following multiplication by a $0.3 \mathrm{~Hz}$ exponential function.

Spectra were processed (phased and baseline corrected) using the ADC/Spec Manager (Version 9.15) software. The phase and the baseline of the spectra were manually corrected. Integration of peaks was processed for regions $0.20-4.20 \mathrm{ppm}$ and $5.08-9.60 \mathrm{ppm}$, excluding the water resonance, with an integral width of $0.04 \mathrm{ppm}$, producing 213 integral buckets in total. All spectra were normalised to total signal. The data were analysed using principal components analysis (PCA) and partial least square discriminant analysis (PLS-DA) (see below).

\subsection{Blood plasma lipid extraction}

Metabolite extraction was achieved using methanol, chloroform and water based on the method of Bligh and Dyer (Bligh and Dyer 1959). Briefly, $150 \mu \mathrm{l}$ of blood plasma were mixed with $600 \mu$ methanol: chloroform (2:1). Samples were sonicated for $15 \mathrm{~min}$. Water and chloroform were added (200 $\mu \mathrm{l}$ of each) and the samples centrifuged for $20 \mathrm{~min}$. The resulting aqueous and organic layers were separated from the protein pellet. The organic layer was dried overnight in a fume food whilst the aqueous extracts were dried using an evacuated centrifuge (Eppendorf, Hamburg, Germany).

\section{$2.4{ }^{1} \mathrm{H}-\mathrm{NMR}$ spectroscopy of aqueous blood plasma extracts}

The dried extracts of the aqueous phase were rehydrated in $600 \mu \mathrm{l}$ of $\mathrm{D}_{2} \mathrm{O}$, buffered with $15 \mathrm{mM}$ sodium phosphate
(pH 7.4) containing $1 \mathrm{mM}$ sodium-3-(tri-methylsilyl)2,2,3,3-tetradeuterio-propionate (TSP; Cambridge Isotope Laboratories, Andover, MA) as an internal standard. The samples were analysed using the pulse sequence, NMR spectrometer, magnet and probe described above. Spectra were collected across 128 transients into $32 \mathrm{k}$ data points and with a $2 \mathrm{~s}$ inter-pulse relaxation delay. The spectra were processed using the method described above for blood plasma.

\subsection{Gas chromatography—mass spectrometry (GC-MS)}

Lipids were extracted from the plasma sample using a Folch extraction to prepare fatty acid methyl esters (Folch et al. 1957). One millilitre of methanol, containing $100 \mu \mathrm{g} / \mathrm{ml}$ undecanoic acid (C11:0), was added to $200 \mu \mathrm{l}$ sample. The contents were vortex mixed for $1 \mathrm{~min}$ at $1,000 \mathrm{rpm}$, before adding $2 \mathrm{ml}$ of chloroform. The contents were mixed, as before, for a further minute. $1 \mathrm{ml}$ of $2 \%$ potassium chloride was added and the contents were mixed as previously described. The test-tube was transferred to a centrifuge for 5 $\min$ at $2,000 \mathrm{rpm}$ at a temperature of $4^{\circ} \mathrm{C}$. The lower chloroform layer was removed to a $12 \times 75 \mathrm{~mm}$ glass tube and evaporated to near dryness in a vacuum concentrator at $45^{\circ} \mathrm{C}$ for $15 \mathrm{~min}$. The residue was reconstituted in $3 \mathrm{ml}$ of methanol saturated with sodium hydroxide (a concentration of $\sim 1 \mathrm{~mol} / \mathrm{l}$.) The contents were transferred to a $25 \mathrm{ml}$ tube, with stopper, and heated at $70^{\circ} \mathrm{C}$ in a water bath for 30 min. After a brief period of cooling, to condense toxic vapor, $4 \mathrm{ml}$ of methanol containing $14 \%$ boron trifluoride was added, and the contents heated at $70^{\circ} \mathrm{C}$ for a further 
$20 \min .1 \mathrm{ml} n$-hexane was added to the tube to extract the derivatized fatty acids (Morrison and Smith 1964). Fifteen millilitres of de-ionized water was added to the test-tube, to stop the reaction. The test-tube was left for a short time to allow the contents to separate into discreet layers. The upper $n$-hexane layer was removed to a fresh $12 \times 75 \mathrm{~mm}$ glass tube, containing a little anhydrous sodium sulphate, as drying agent.

Samples were then injected into a Shimadzu GC-17AAF gas chromatograph (Shimadzu Corporation, Kyoto, Japan), fitted with a SGE BPX70 column, (SGE International, Australia) using a programmed temperature vaporisation method. Samples were maintained at $55^{\circ} \mathrm{C}$ for $1 \mathrm{~min}$, then increased to a temperature of $170^{\circ} \mathrm{C}$ at a rate of $14^{\circ} \mathrm{C} / \mathrm{min}$, then to $220^{\circ} \mathrm{C}$ at $3.5^{\circ} \mathrm{C} / \mathrm{min}$, where it was held for $1 \mathrm{~min}$. A chromatogram was then obtained. Concentrations were calculated using the internal standard (C11:0) recovery and measured against a multi level calibration curve, plotted for each quantified fatty acid methyl ester using Shimadzu VP-software. The table containing the levels of 26 fatty acids for all samples was used to perform PCA and PLSDA. As absolute values were used no normalisation was necessary.

\subsection{Liquid chromatography-mass spectrometry (LC-MS)}

Lipid extracts were re-dissolved in 2:1 methanol:chloroform and injected into a UPLC Acquity ${ }^{\mathrm{TM}}$ (Waters, Milford, USA) with a C8 column (ACQUITY UPLC ${ }^{\mathrm{TM}} \mathrm{BEH}$ C8 $2.1 \times 100 \mathrm{~mm}, 1.7 \mu \mathrm{m}$, Waters). Mobile phase A was made up of water with $0.1 \%$ formic acid and $10 \mathrm{mM}$ ammonium acetate, while phase B was acetonitrile:isopropanol $(5: 2)$ with $0.1 \%$ formic acid and $10 \mathrm{mM}$ ammonium acetate. The analytes were eluted from the column over 10 min with a gradient of $60-100 \%$ B followed by 2 min at $100 \% \mathrm{~B}$ and 2 min of column re-equilibration at the starting condition $60 \% \mathrm{~B}$. The column flow rate was $600 \mu \mathrm{l} / \mathrm{min}$. The UPLC was coupled to a Q-ToF micro (Waters, Milford, USA) with a 1:1 eluent split. After every 10 sample injections a pooled sample followed by a blank were injected in order to ensure consistent performance of the system. The randomised sample list of extracts was run three times, resulting in the repeating measurements being a few days apart. MassLynx (Waters, Milford, USA) was used to export spectra as ASCII files and further processing was done using in-house software written in the package IGOR PRO (WaveMetrics, Lake Oswego, Oregon, USA). Every LC-MS file was compressed to the mass spectral dimension by adding all retention time points at a given $\mathrm{m} / \mathrm{z}$ value. The resulting, summed mass spectra were aligned to correct for mass drifts over the days of the analysis. Every data point in the spectrum was kept as a variable in the following SIMCA-P + 11 (v11.0, Umetrics, Umea, Sweden) analysis if it exceeded the noise threshold in more than $10 \%$ of all the spectra from all samples. This left 1,745 data points for each spectrum to be analysed by PLS-DA. The spectral data for each sample was normalised to total ion count. Re-analysis of the raw data ensured that the extracted ion chromatograms of the significant loadings displayed only one peak and was not a combination of different compounds. Typically the average coefficient of variation for LC-MS measurements was $\sim 10 \%$ of the mean as determined by repeated injections of the pool sample examined throughout the study. As expected, the values for specific lipids were below this value for high intensity peaks and higher for low abundant signals. Lipids were identified by a combination of retention time, mass and MS/MS measurements. No internal standard was used, as only relative changes were studied.

\subsection{Multivariate data analysis of metabolomic data}

For each analytical assay the resulting data table was imported into SIMCA-P (v11.0, Umetrics, Umea, Sweden) for multivariate analysis by PCA and PLS-DA (an extension of PCA used for classification). Data was normalised to total intensity (except GC-MS) and Pareto scaled. Identification of major metabolic perturbations within the pattern recognition models was achieved by analysis of corresponding loadings plots. The parameters $R^{2}$ and $Q^{2}$ were used as measures for the robustness of a pattern recognition model. $R^{2}$ is the fraction of variance explained by a component, and cross validation of $R^{2}$ gives $Q^{2}$ which reveals the fraction of the total variation predicted by a component, it is a measure for the difference between two (or more) groups. Typically a robust model has $R^{2}>50 \%$ and $Q^{2}>40 \%$.

For the LC-MS data the sections of the mass spectra that had been cut out for the analysis were filled with zero values in order to display the loadings resembling a differential mass spectrum for a more intuitive viewing. The peaks that contributed significantly to the separation as determined by the loading were then viewed as raw spectra in order to find the retention time of the mass that changed. Only in a few cases, were there more than one retention time for a specific ion, and even for these cases, the peak responsible for classification could be identified by visual inspection of raw chromatograms.

\section{Results}

Independent of whether the samples were measured by NMR spectroscopy, GC-MS or LC-MS, the ability to distinguish between the groups shown in Fig. 1 followed the 
same general pattern. The biggest differences in the plasma profiles were associated with the intake of fish oil. The MS based methods, showed some more subtle changes associated with weight loss. The results obtained are discussed in detail below for the three analytical tools used.

\subsection{High resolution ${ }^{1}$ H-NMR spectroscopy}

The dominant trend in the total NMR data set was associated with the degree of saturation of the total lipid fraction. All the samples collected from women taking fish oil had an increase in the resonance intensity at $5.24-5.32 \mathrm{ppm}$, corresponding to $\underline{\mathrm{C}}=\mathrm{C} \underline{\mathrm{H}}$, and $2.72-2.80$ corresponding to $\mathrm{CH}=\mathrm{CH}-\mathrm{C}_{\underline{1}}-$ $\mathrm{CH}=\mathrm{CH}$ moieties. Equally, the integral regions between 1.24 and $1.32 \mathrm{ppm}$, where the $\mathrm{CH}_{2}-\mathrm{CH}_{2}-\mathrm{CH}_{2}$ resonances associated with saturation in fatty acids contribute to the spectra, were reduced in the fish oil groups relative to all other groups (Fig. 2). No differences associated with weight loss, could be found in the plasma NMR dataset.

As the lipid resonances overlap with aqueous metabolites, for example the 1.24-1.32 ppm region overlaps with a doublet from lactate, we examined aqueous extracts of the blood plasma to examine whether differences associated with aqueous metabolites and not lipid species were responsible for the classifications. However, analysis of the entire dataset demonstrated no separation for any of the groups, indicating that all classifications were associated with lipid species (data not shown). This conclusion held for all regions of the NMR spectrum where resonances from low molecular weight metabolites were obscured by broad lipid resonances. Thus, future analysis focussed on the lipid fraction.

\subsection{GC-MS of total fatty acid content of blood plasma}

As the NMR data suggested that the largest differences between the groups are associated with lipid moities, in the next stage of the analysis, the total fatty acid profiles were measured as fatty acid methyl esters (FAMEs) using GC-MS. This data has been previously presented using a univariate statistical approach (Krebs et al. 2006). Two way comparisons were made between the groups using pattern recognition methods. The most robust PLS-DA models of the data were built between IFO or FFO and any other group, indicating that fish oil supplementation dominated the measured changes. However, more subtle
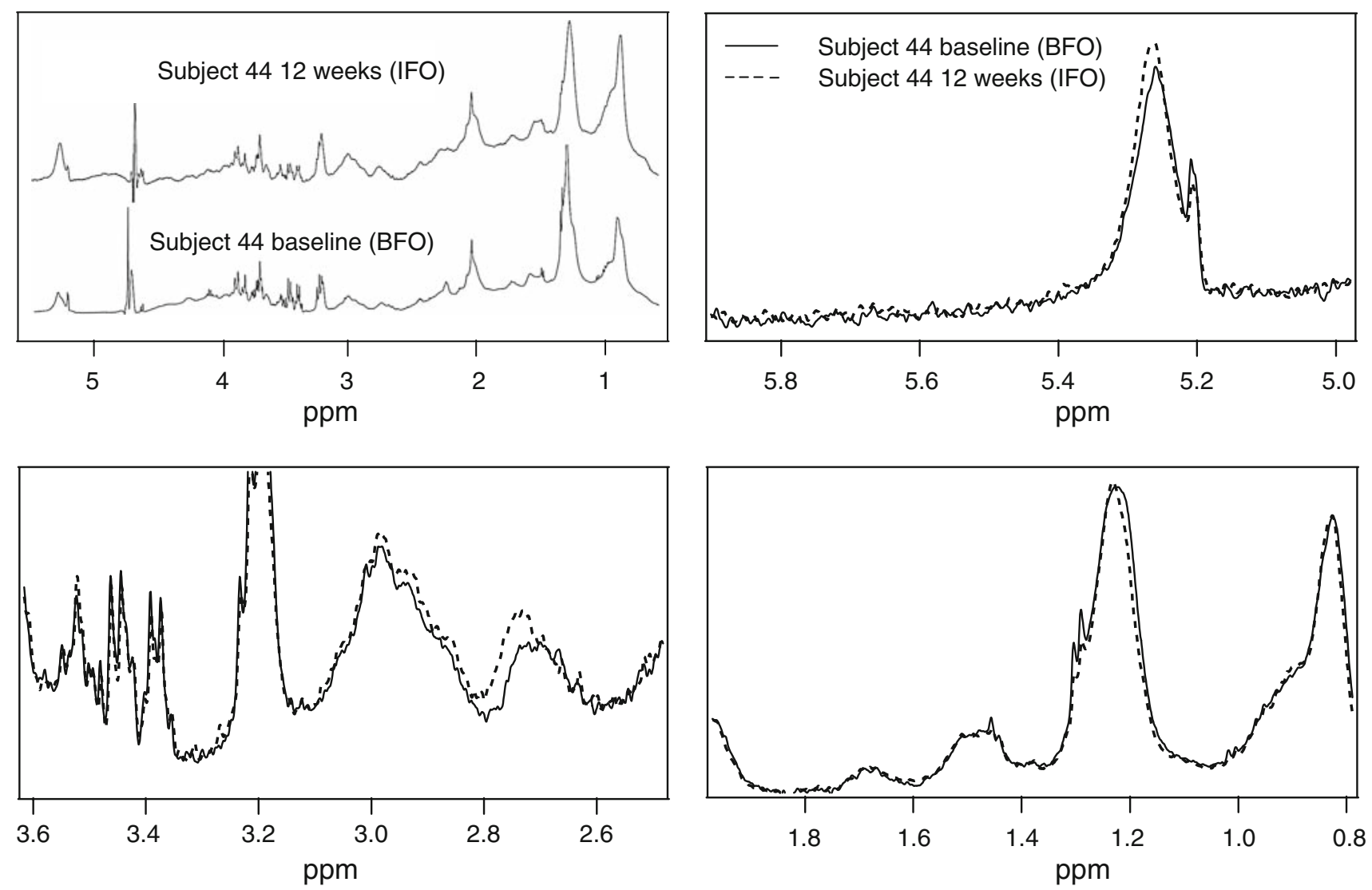

Fig. 2 Two NMR spectra of a subjects' plasma at baseline (without fish supplementation; BFO) and after 12 weeks (with fish oil supplementation; IFO). The increasing number of double bonds in the lipid signals was the only clear change in the NMR spectra of the plasma 
differences due to weight loss could be detected between IPO or IFO and IC. The robustness $\left(Q^{2}\right)$ was used to assess the validity of the models. While models with a $Q^{2}>0.08$ are considered statistically significant, only those with $Q^{2}>0.4$ robustly predict class membership for individual samples left out of the model. In these comparisons BFO/ IFO had a $Q^{2}$ of 0.63 , while BPO/IPO had a $Q^{2}$ of only 0.53 , demonstrating that feeding fish oil had the biggest impact on the lipid profile while weight loss produced a less robust model.

To understand the metabolic changes that were responsible for these classifications the loadings of the PLS-DA plots for the GC-MS data were analysed. Figure $3 \mathrm{a}$ shows the composition of the fish- and placebo oil used in this study, with the fats administered as triacylglycerols (TAGs), while Fig. 3b shows a typical FAME profile from a subject at baseline. The most abundant fatty acids in plasma are 16:0, 18:1n-9c and 18:2n-6c and these are also the main components of the placebo oil, in order to have the smallest overall effect on the lipid profile in the group receiving the placebo oil. The fish oil supplementation resulted in significant increases in the fatty acids supplied in the supplement in the FO group (Fig. 3c, d; Table 2). However, the fatty acids $\mathrm{C} 22: 5$ (both $\mathrm{n} 3$ and $\mathrm{n} 6$ species) were affected by both weight loss (decreased in
Table 2 Significant changes in FAMEs, with the intervention cause and direction of the changes

\begin{tabular}{ll}
\hline Fatty acid & Change in dataset \\
\hline C16:1n-9c & Reduced with fish oil supplementation \\
C18:1n-9c & \\
C18:3n-3 & \\
C20:3n-3 & \\
C20:5n-3 & Increased with fish oil supplementation \\
C22:6n-3 & \\
C22:5 & \\
C22:5 & Reduced with weight loss at 12 \& 24 weeks \\
C20:1n-9 & Reduced with weight loss at 12 weeks \\
C18:1n-7c & Increased with weight loss (in placebo group only)
\end{tabular}

IPO vs. BPO) and fish oil supplementation (increased in IFO vs. BFO). While the fatty acid was the third most abundant fatty acid constituent in fish oil (Fig. 3a), weight loss had a greater effect, and hence an overall (but smaller) decrease was seen between BFO and IFO compared with $\mathrm{BPO}$ and IPO. However, the seemingly significant change of 10:0 (Fig. 3d) was discarded because it was too close to the detection limit to be reliably quantified.
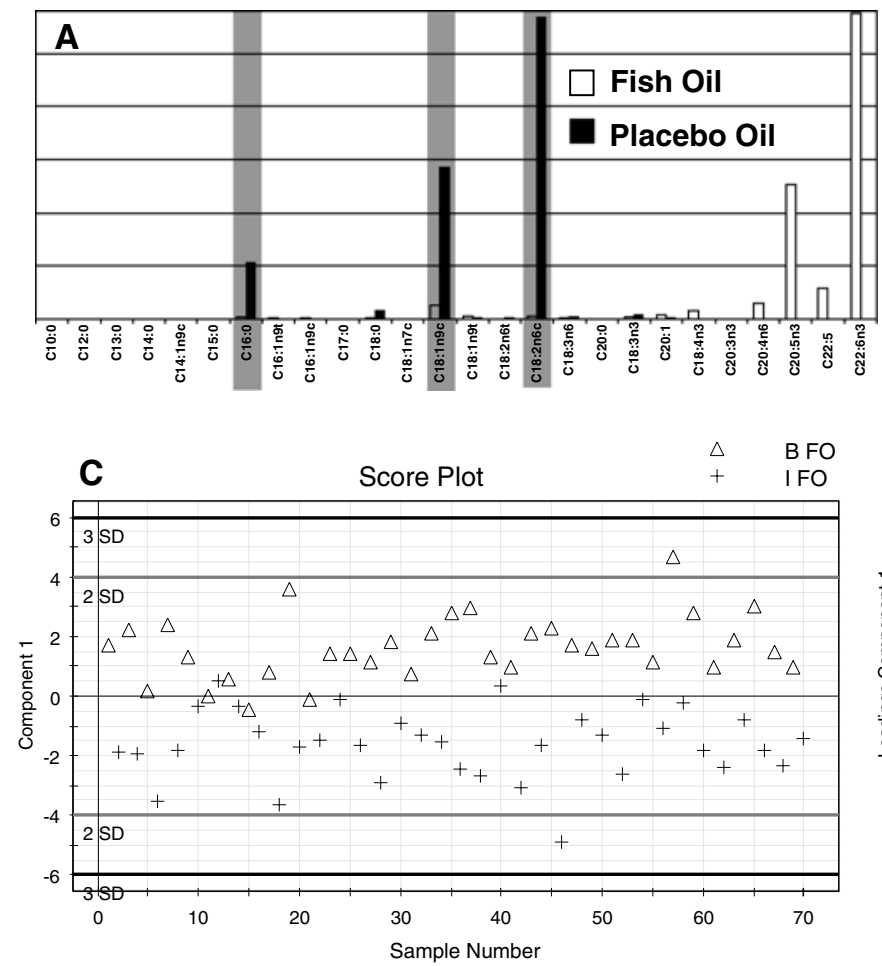

Fig. 3 Fatty acid methyl ester (FAME) analysis with the most common fatty acids highlighted in panels A, B and D. a Fatty acid composition of the fish ( $\square$ - - and placebo oil ( $\square$ ) capsules measured by GC-MS. b Typical FAME profile of blood plasma from a subject.
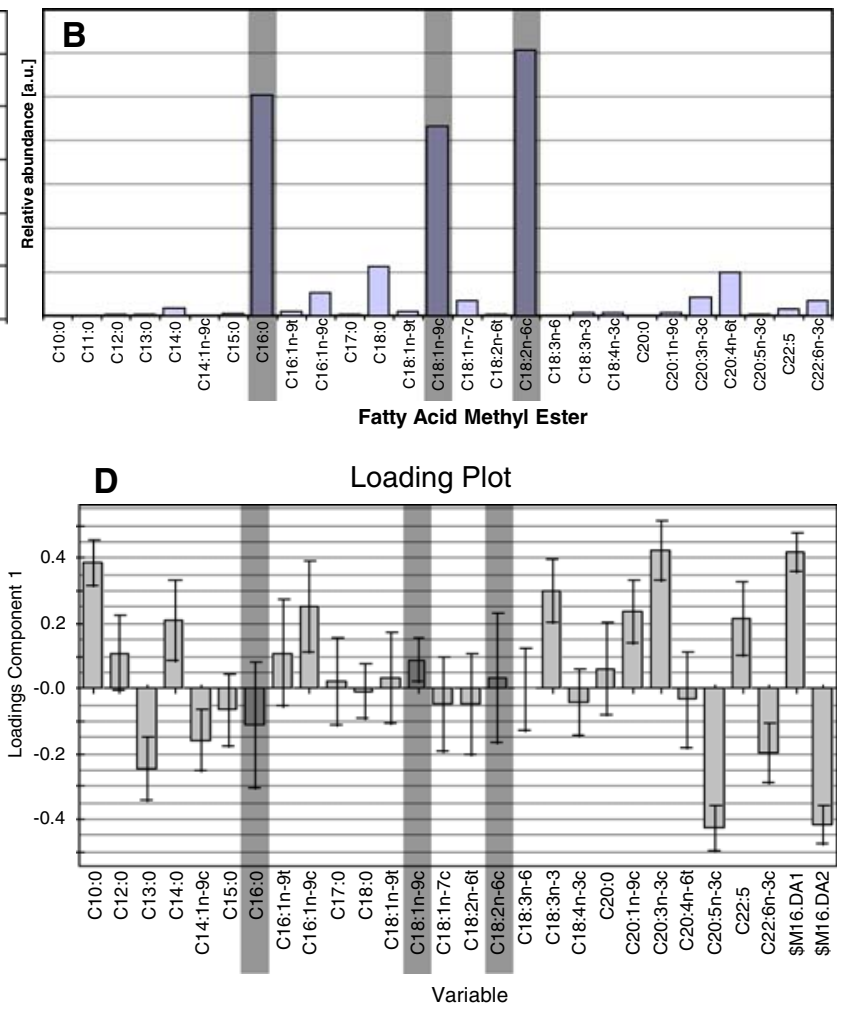

The shown sample is from the BPO group. c Score plot of PLS-DA model between the baseline and 12-week time point of the fish oil group (BFO vs. IFO; $Q^{2}=0.63, R^{2}=0.68$. d Loading plot driving the separation shown in $\mathbf{c}$ 


\subsection{LC-MS}

One limitation of understanding fatty acid metabolism through GC-MS is that the approach measures total fatty acid content, rather than what lipid species might contribute to these changes. LC-MS was performed to address this question. Figure 4 illustrates a typical 2D LC-MS run (a) and the reproducibility of the method used (b). The peaks at higher mass and later retention time are from the triacylglycerides (TAGs) while the cluster of peaks at earlier retention time and lower mass are from the polar phospholipids (a). The chromatography time used in these runs was a compromise between the need to resolve as large a number of lipid species during the assay and a need to minimise the run time to allow data acquisition in a reasonable timescale. Panel b demonstrates the reproducibility of the LC-MS measurements with a subset of spectra from the BPO group. A subset of the samples from the BPO group, each injected three times, are plotted in a PCA scores plot. Repeats of every sample were given the same symbol, demonstrating that the biological variation is clearly greater than the variation between technical repeats.

From both visual inspection and pattern recognition analysis, as with the GC-MS data, the main differences in the lipid profile was due to the fish oil supplementation and only more subtle differences were found due to weight loss. A PLS-DA scores plot of all 3 groups at the intermediate time point show minor overlap between the IFO and IPO/ IC groups and major overlap between the IPO and IC groups (supplementary Fig. 1).

To better define the metabolic differences between groups two-way comparisons for all groups were carried out using PLS-DA. Figure 5 shows an example of a PLSDA model $\left(Q^{2}=0.85 ; R^{2}=0.90\right.$ - the highest $Q^{2}$ of all 2-way models) between IFO and BPO with scores (panel a) and the loadings (panel b) plot including the standard deviation of the loadings (grey lines). The $Q^{2}$ measure was used to determine how dissimilar two groups were across the whole dataset. Figure 6 summarises all possible pair wise comparisons for the LC-MS dataset by PLS-DA. The most robust models, and hence largest biochemical differences, between groups were associated with fish oil supplementation (any group vs. IFO and FFO). However, more minor effects were associated with short term weight loss, and no significant differences (within our definition of model robustness) were found for the control group (BFO vs. IFO $Q^{2}=0.72$; BPO vs. IPO $Q^{2}=0.40 ; \mathrm{BC}$ vs. IC $\left.Q^{2}=0.15\right)$.

The PLS-DA plots were validated by randomly permutating the value of $Y$ and comparing the $Q^{2}$ to the true value. In the case of the comparison between BC and IPO $\left(Q^{2}=0.5\right)$ the resulting regression line through the true and false $Q^{2} \mathrm{~s}$ has an offset of -0.3 demonstrating the robustness of the model (supplementary Fig. 2). Similar results were achieved with all models considered significant $\left(Q^{2}>0.4\right)$ ensuring there was no over fitting of the data.

Further confidence in the models was gained because each mass spectral peak consists of several data points creating redundancy for each lipid species. These multiple data points consistently were identified together in the loadings plots (Fig. 5b). The lipids responsible for classification above a set threshold (eight times the noise level) and with error bars $< \pm 40 \%$ were analysed in more detail using a combination of tandem MS, retention time and database searching. Careful evaluation of all comparisons yielded a list of signals that change significantly and could be attributed either to fish oil intake or diet intervention (Table 3).

In terms of the class of lipid species affected the largest decreases on fish oil supplementation were associated with TAGs, suggesting that this class of lipids had decreased in general, which has been published before (Harris 1996; Krebs et al. 2006). This was confirmed by biochemical assay for total TAG concentration (e.g. $P=5.13 \times 10^{-4}$ for BFO and IFO comparison, according to a Student $t$-test). However, some of the high intensity TAG signals, such as 52:3 and 52:2, in the raw data did not change significantly with fish oil intake indicating a remodelling of this class of lipid as well as an overall reduction. following fish oil feeding.

\section{Discussion}

In this study we have employed a combined NMR spectroscopy, GC-MS and LC-MS metabolomic analysis of blood plasma in order to identify metabolic changes associated with fish oil supplementation, weight loss and the interaction between these two interventions. Currently no single analytical tool will provide a complete analysis of the metabolome and our strategy for open profiling is to use a combination of analytical devices. Although being the technique of least sensitivity, ${ }^{1} \mathrm{H}-\mathrm{NMR}$ spectroscopy was both rapid $(\sim 12 \mathrm{~min}$ per sample) and reproducible. However, because of the chemical nature of lipid species ${ }^{1} \mathrm{H}-\mathrm{NMR}$ spectroscopy provides poor discrimination between individual fatty acids. However, GC-MS provides a robust method for distinguishing changes in total fatty acid concentration in samples, but analysis times are longer $(\sim 30 \mathrm{~min})$ and require prior derivatisation. LC-MS is capable of examining intact lipids directly. While assay times in this study were $\sim 15$ min per sample, the lower reproducibility of this approach compared with the other two analytical tools necessitated the analysis of samples in triplicate. 

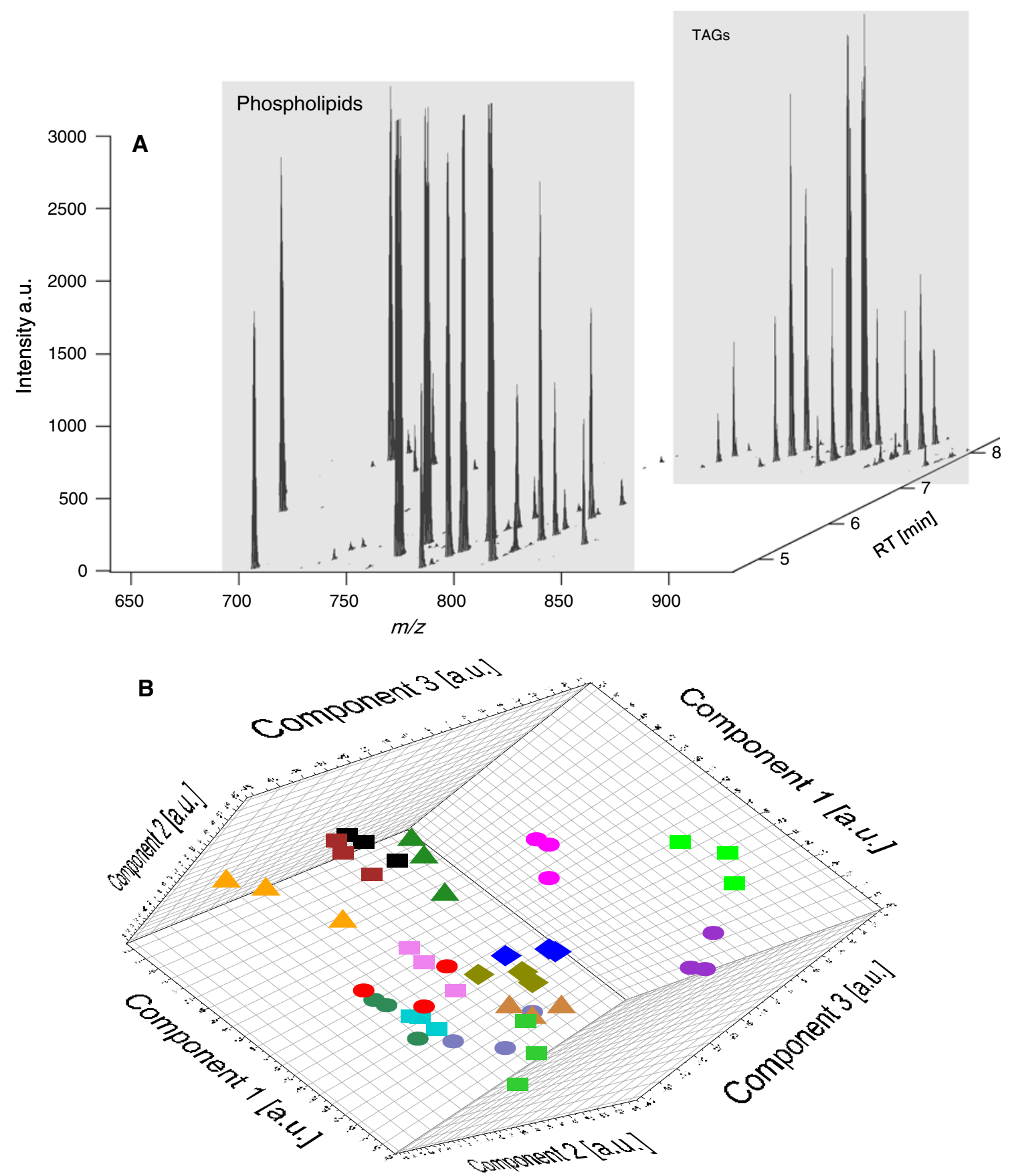

Fig. 4 a Example of the LC-MS measurement of a lipid extract from plasma. b PCA plot demonstrating the reproducibility of the LC-MS data. Each symbol represents a sample from the baseline of the placebo oil group and was injected three times. The technical repeats

GC-MS is a robust method for fatty acid analysis and is often used for metabolomics. It does however, when applied to lipid analysis, only measure the total fatty acid composition. The information on the origin of the fatty acids is lost during the derivatisation process. LC-MS on the other hand measures intact lipid species but the information on the exact fatty acid composition is harder to cluster together indicating that the variation between the samples is much bigger than the technical variation even if the samples were acquired over several days

determine. However, both techniques may be used complimentarily to maximise the information obtainable from the lipid fraction.

The data obtained by NMR spectroscopy demonstrated two important pieces of information that determined the direction of the mass spectrometric analyses. Firstly, no difference was found between samples for the aqueous 


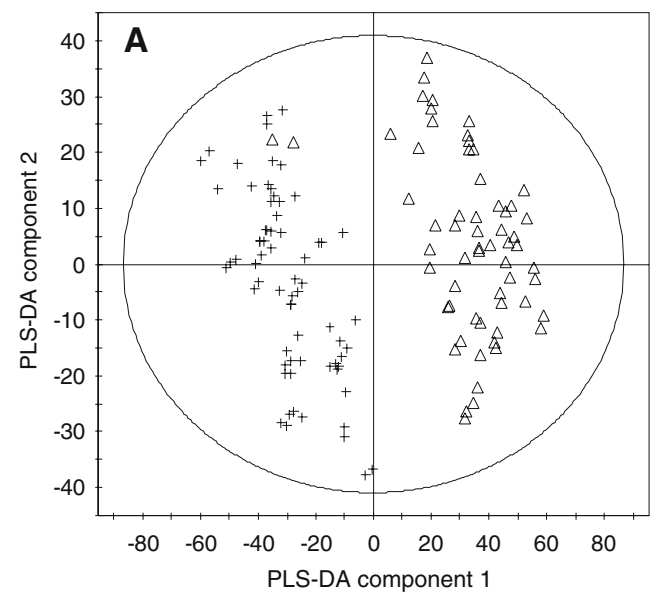

Fig. 5 a Scores plot of a PLS-DA model $\left(Q^{2}=0.85 ; R^{2}=0.90\right)$ between IFO $(\Delta)$ and BPO $(+)$. b Coefficients driving the separation in A displayed as a spectrum. The Black dots are the variable loadings while the grey lines represent the error bars. Multiple variables for

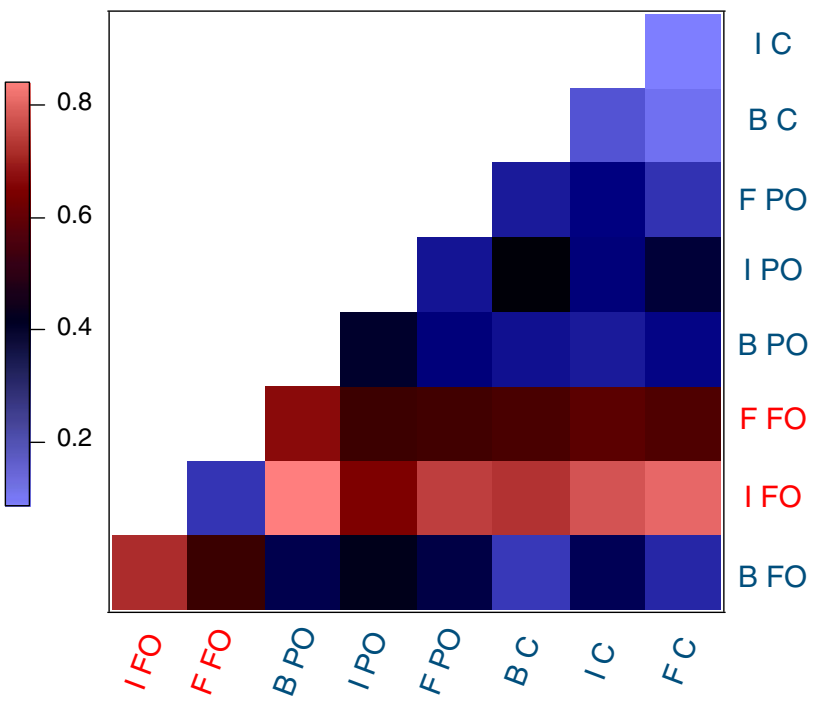

Fig. $6 Q^{2}$ of all pairwise comparisons between the nine groups of the study. Note the fish oil is most different from all other groups. $\mathrm{I}=$ intermediate, $\mathrm{B}=$ baseline, $\mathrm{F}=$ final timepoint; $\mathrm{C}=$ control, $\mathrm{PO}=$ placebo oil, $\mathrm{FO}=$ fish oil

metabolites but clear differences were detected for the whole plasma containing lipids. Secondly, the differences in the plasma lipids were, not surprisingly, associated with the degree of saturation in the fats of the groups supplementing their diet with fish oil. As there is significant resonance overlap between different fatty acids/lipid species further analyses were performed by mass spectrometry to determine the biochemical changes associated with fish oil supplementation.

The total reduction of TAGs upon increased intake of n3 PUFAs that was found in these data is well established in the literature (Harris 1996; Krebs et al. 2006). However, we each peak indicate that the whole signal is changing. The horizontal black lines were used as a threshold and loading higher that that were considered significant

demonstrate in the present study that a remodelling of the TAG species has occurred. While the majority of the TAG(48: $x$ where $x$ signifies the number of double bonds) and $\operatorname{TAG}(50: x)$ decreased in concentration, the low abundance TAG(46:x) and TAG(56:x) and the high abundance TAG(52:x) and TAG(54:x) do not, with the exception of TAGs containing five unsaturated bonds across all three fatty acids.

The diverse range of changes detected in the lipid profile by LC-MS confirm previous literature reports where others have used LC-MS to study either animal models or patients with obesity, type 2 diabetes and the metabolic syndrome. Yetukuri and colleagues used LC-MS to study liver tissue of ob/ob mice and compared this to tissue from wild type animals (Yetukuri 2007). The authors found a general increase of short chain TAGs in ob/ob but not long chain ones. Specifically, TAG(48:2), TAG(48:1) and TAG(50:2) are decreased with fish oil supplementation in our study and increased in ob/ob mouse liver lipid profiles (Yetukuri 2007). Another study from the same group (Medina-Gomez 2007) identified a major decrease of TAG(48:2) in the POKO mouse (a double gene deletion of the leptin receptor and PPAR $\gamma$ ) and a minor decrease in white adipose tissue (WAT) from ob/ob mice. In skeletal muscle TAG(48:2) and TAG(48:1) were decreased in the POKO mouse but not in ob/ob. All of these mentioned TAGs were also found to be altered in our study, demonstrating that the triglyceride pool is selectively modulated by obesity, type 2 diabetes and dietary intervention.

Another study, where subjects with metabolic syndrome were subjected to a 33-week diet intervention study, has shown a similar rearrangement of chain length in plasma TAGs (Schwab et al. 2008). The authors demonstrate a 
Table 3 Summary of significant changes in LC-MS peaks between groups

\begin{tabular}{|c|c|c|c|}
\hline & Loading mass $\left(\mathrm{M}+\mathrm{H}^{+}\right)$ & RT & ID \\
\hline \multirow[t]{10}{*}{ Increased with fish oil supplementation } & 688.8 & 7.56 & DAG(40:10) \\
\hline & 780.7 & 4.83 & $P S(36: 6)$ or $P C(36: 5)$ \\
\hline & 806.6 & 5.04 & $\mathrm{PC}(38: 6)$ \\
\hline & 808.7 & 5.24 & $P S(36: 6)$ or $P C(36: 5)$ \\
\hline & 813.7 & 6.22 & $\operatorname{SM}(38: 2)$ \\
\hline & 834.7 & 5.49 & $P S(40: 7)$ or $P C(40: 6)$ \\
\hline & 920.8 & 7.72 & $\mathrm{TAG}(56: 8)$ \\
\hline & 894.8 & 7.66 & $\operatorname{TAG}(54: 7)^{\mathrm{a}}$ \\
\hline & 731.8 & 5.31 & $P A(38: 1)^{\mathrm{a}}$ \\
\hline & 714.8 & 7.61 & $\operatorname{DAG}(42: 11)^{\mathrm{a}}$ \\
\hline \multirow[t]{11}{*}{ Decreased with fish oil supplementation } & 784.7 & 5.31 & $\mathrm{PC}(36: 3)$ \\
\hline & 812.7 & 5.76 & $\mathrm{PC}(38: 3)$ \\
\hline & 820.9 & 7.84 & TAG(48:2) \\
\hline & 822.9 & 7.99 & TAG(48:1) \\
\hline & 846.9 & 7.85 & TAG(50:3) \\
\hline & 848.8 & 8.01 & TAG(50:2) \\
\hline & 850.9 & 8.16 & TAG(50:1) \\
\hline & 870.9 & 7.72 & TAG(52:5) \\
\hline & 898.9 & 7.91 & TAG(54:5) \\
\hline & 732.8 & 5.32 & $P S(32: 2)$ or $P C(32: 1)^{\mathrm{a}}$ \\
\hline & 794.9 & 5.49 & $P E(40: 5)^{\mathrm{a}}$ \\
\hline \multirow[t]{2}{*}{ Increased with weight loss (WLPO only) } & 703.7 & 4.78 & $\operatorname{SM}(30: 1)$ \\
\hline & 766.8 & 5.38 & $P E(38: 5)$ \\
\hline Decreased with weight loss only & 878.8 & 8.31 & $T A G(52: 1)$ \\
\hline
\end{tabular}

Note: Tentative (low abundance/no MS/MS) or ambiguous identifications are in italics

$T A G$ triacylglycerol, $D A G$ diacylglycerol, $P C$ glycerophosphatidyl choline, $P S$ glycerophosphoserine, $P E$ glycerophospho ethanolamine, $P A$ glycerophospatydic acid, $S M$ sphingomyeline

${ }^{\text {a }}$ Denotes signals at very low abundance in the raw data

decrease in short chain saturated fatty acids and that this change is correlated to insulin sensitivity. Despite the low sample size this study did benefit from a wider lipidomic coverage, probably rendering the analysis more sensitive to lipid remodelling. However our study showed clearly that weight loss with the addition of dietary fish oil supplementation causes a large rearrangement of the lipid triglyceride pool.

The complimentary nature of a combined GC-MS and LC-MS approach was evident from the cross correlation of the two data sets. For example, the decrease in the fatty acids $18: 3$ and 20:3 with fish oil supplementation as determined by GC-MS is consistent with the decrease of $\mathrm{PC}(36: 3)$ and $\mathrm{PC}(38: 3)$ if one assumes that one of the fatty acids in these species is the highly abundant palmitate (16:0 — Fig. 3b). Neither of these fatty acids correlates with any TAG reduction indicating that the decrease in 18:3 and 20:3 fatty acids preferentially affect PC lipids rather than triglycerides. This suggests that LC n-3 PUFA increase the metabolism of short chain n-3 PC.
As shown by the LC-MS data, the wide variety of lipid types (phospholipids and TAG/DAG) that increase with fish oil supplementation all have a high degree of unsaturation. This is most likely due to the effect of lipid synthesis with the supplemented n-3 PUFA. Unfortunately, one complication in interpreting the results produced in this study is that it was not possible to discriminate between effects associated with fish oil supplementation alone and the combined action of fish oil and weight loss as the study design did not include a group supplemented with fish oil in the absence of weight loss (Krebs et al. 2006). The inclusion of this group would have allowed separation of the weight loss and fish oil effects. Hence, it remains unclear as to which of the changes are due to the fish oil intake alone and which of them may be due to a synergistic weight loss/fish oil supplementation effect.

One of the lipids with a relatively high degree of saturation which increased in concentration in the fish oil supplemented group was $\mathrm{SM}(38: 2)$. Conversely the concentrations of $\operatorname{SM}(42: 1)$ and $\operatorname{SM}(42: 2)$ have been found to 
decrease in ob/ob mouse liver (Yetukuri 2007). Thus, this increase in SM may be a beneficial effect of fish oil feeding although the exact mechanism remains to be elucidated underlying the complexity of the data set obtained by LC-MS lipidomics.

The changes observed based on weight loss alone include the increase in concentration of $\operatorname{SM}(30: 1)$ containing very short chained fatty acids. In addition a decrease in TAG(52:1) was detected. This might suggest a decrease in specific TAGs rather than the general reduction of short chain species we observe with the fish oil supplemented groups. However, these were some of the few biological changes associated with weight loss and placebo oil detected across this study, in part because of the study design and the very large changes associated with dietary intervention which dominate the dataset.

The chromatogram peak representing fatty acid 22:5 (a sum of $\mathrm{n}-6$ and $\mathrm{n}-3$ fatty acids) displayed an interesting behaviour as determined by GC-MS. Despite it being the third most abundant fatty acid in the fish oil supplementation (Fig. 3a) it decreased in the comparison between BFO and IFO (Fig. 3c, d) after fish oil supplementation. However, this fatty acid decreased in the weight loss group at 12 and 24 weeks with higher significance as determined by the relative intensity of the loadings. This leads to the conclusion, that weight loss decreases $22: 5$ but that this reduction is masked slightly by the supplement of fish oil in the diet, so that although weight loss results in an overall decrease between the BFO and IFO group this is not as large as that detected between BPO and IPO. The decrease in the fatty acid is also reflected in the decrease of serum TAG(54:5) which might well be made up of 2 16:0 (one of the most common fatty acids-Fig. 3b) and one 22:5 fatty acids. TAG(54:5) therefore is an exception of a decreasing serum TAG with more than 52 carbons. Interestingly, TAG(54:5) has been reported to decrease in WAT in ob/ob mice and increase in the double knock out POKO in the same tissue as compared to wild type (Medina-Gomez 2007). Assuming that TAG(54:5) contains fatty acid 22:5 this means there is an inverse correlation of the amount of 22:5 in WAT and serum. 22:5 is synthesised from 18:2. The last step of the synthesis takes place in the peroxisomes (Innis 2003; Innis et al. 2004). The decrease in ob/ob WAT but increase in the POKO mouse suggests that PPAR $\gamma$ promotes the mobilisation of $22: 5$ from adipose tissue. In respect to the study investigated here this suggests that weight loss increases the PPAR $\gamma$ activity in WAT cells.

This result also illustrates the difficulty of ultimately identifying the exact lipid composition in the samples. This holds particularly true for low abundance lipid species. The number of structural isomers within lipids is enormous and the reliable separation and quantification of all the lipid species is in the centre of active, ongoing research. With time this will lead to more assertive assignments and ultimately improved biological interpretation of disease processes in the future.

Surprisingly there were also detectable differences between groups that were planned to be similar. For instance the two baseline groups BPO and BFO (Fig. 6) have a relatively high $Q^{2}$ of almost 0.4 . This indicates the difficulty of randomising people even with $\sim 30$ subjects in each group. Furthermore, such studies are also affected by human behaviour. Being part of a study as a control group is most likely going to influence the behaviour of the control group subjects. Indeed we would expect this effect to be most pronounced before samples are taken. This suggests that blood plasma based studies should perhaps be considered alongside material with a slower changing profile such as red blood cells or adipose tissue.

\section{Conclusions}

This study investigated fish oil supplemented diet in combination with weight loss by a variety of metabolomic methods. The fish oil supplementation was found to have the largest effect on plasma metabolic profiles. Furthermore only the lipid fraction seemed to be affected as was determined by high resolution ${ }^{1} \mathrm{H}-\mathrm{NMR}$ spectroscopy. Fish oil supplementation in conjunction with weight loss was found to reduce the total serum TAG content by selectively reducing the short chain fatty acids. Weight loss caused a reduction in the fatty acid 22:5, although this fatty acid was also increased by fish oil supplementation relative to individuals receiving placebo oil. Overall, these results demonstrate the selective modulation of blood plasma TAGs by fish oil supplementation.

Acknowledgments This work was supported by the United Kingdom NIHR Cambridge Biomedical Research Centre. In addition GM and JLG were supported by the British Heart Foundation, the Medical Research Council and the Royal Society, UK. JLG \& SAJ are members of the Medical Research Council Centre for Obesity and Related Metabolic Diseases (MRC CORD), and an affiliate member of the University of Cambridge Metabolic Research Laboratories. The authors would like to thank Dr. Richard Knochenmuss for the use of his in-house spectral alignment tool for IGOR, Dr Jeremy Krebs for the original study design and sample collection, and the Nutritional Biochemistry Laboratory at MRC Human Nutrition Research for GCMS analysis.

\section{References}

Bligh, E. G., \& Dyer, W. J. (1959). A rapid method of total lipid extraction and purification. Canadian Journal of Biochemistry and Physiology, 37, 911-917. 
Browning, L. M., et al. (2004). Elevated sialic acid, but not CRP, predicts features of the metabolic syndrome independently of BMI in women. International Journal of Obesity, 28, 10041010. doi:10.1038/sj.ijo.0802711.

Chan, J. M., et al. (1994). Obesity, fat distribution, and weight-gain as risk-factors for clinical diabetes in men. Diabetes Care, 17, 961969. doi:10.2337/diacare.17.9.961.

Colditz, G. A., et al. (1990). Weight as a risk factor for clinical diabetes in women. American Journal of Epidemiology, 132, 501-513.

Folch, J., Lees, M., \& Sloane-Stanley, G. H. (1957). A simple method for the isolation and purification of total lipids from animal tissues. The Journal of Biological Chemistry, 226, 497-509.

Griffin, J. L. (2004). Metabolic profiles to define the genome: Can we hear the phenotypes? Philosophical Transactions of the Royal Society of London. Series B, Biological Sciences, 359, 857-871. doi:10.1098/rstb.2003.1411.

Harris, W. S. (1996). n-3 Fatty acids and lipoproteins: Comparison of results from human and animal studies. Lipids, 31, 243-252. doi: 10.1007/BF02529870.

Hill, A. M., et al. (2007). Combining fish-oil supplements with regular aerobic exercise improves body composition and cardiovascular disease risk factors. The American Journal of Clinical Nutrition, $85,1267-1274$.

Innis, S. M. (2003). Perinatal biochemistry and physiology of longchain polyunsaturated fatty acids. The Journal of Pediatrics, 143, S1-S8. doi:10.1067/S0022-3476(03)00396-2.

Innis, S. M., Vaghri, Z., \& King, D. J. (2004). N-6 Docosapentaenoic acid is not a predictor of low docosahexaenoic acid status in Canadian preschool children. The American Journal of Clinical Nutrition, 80, 768-773.

James, M. J., Gibson, R. A., \& Cleland, L. G. (2000). Dietary polyunsaturated fatty acids and inflammatory mediator production. The American Journal of Clinical Nutrition, 71, 343S348 S.

Knowler, W. C., et al. (2002). Reduction in the incidence of type 2 diabetes with lifestyle intervention or metformin. The New England Journal of Medicine, 346, 393-403. doi:10.1056/ NEJMoa012512.

Krebs, J. D., et al. (2006). Additive benefits of long-chain n-3 polyunsaturated fatty acids and weight-loss in the management of cardiovascular disease risk in overweight hyperinsulinaemic women. International Journal of Obesity, 30, 1535-1544. doi: 10.1038/sj.ijo.0803309.

Kriketos, A. D., et al. (2001). Role of weight loss and polyunsaturated fatty acids in improving metabolic fitness in moderately obese, moderately hypertensive subjects. Journal of Hypertension, 19, 1745-1754. doi:10.1097/00004872-200110000-00007.

Manson, J. A. E., et al. (1990). A prospective-study of obesity and risk of coronary heart-disease in women. The New England Journal of Medicine, 322, 882-889.

Manson, J. E., et al. (1995). Body-weight and mortality among women. The New England Journal of Medicine, 333, 677-685. doi:10.1056/NEJM199509143331101.

Medina-Gomez, G., et al. (2007). PPAR gamma 2 prevents lipotoxicity by controlling adipose tissue expandability and peripheral lipid metabolism. PLOS Genetics, 3(4), e64.

Morrison, W. R., \& Smith, L. M. (1964). Preparation of fatty acid methyl esters and dimethylacetals from lipids with boron fluoride-Methanol. Journal of Lipid Research, 5, 600-608.

Ogden, C. L., et al. (2006). Prevalence of overweight and obesity in the United States, 1999-2004. Journal of the American Medical Association, 295, 1549-1555. doi:10.1001/jama.295.13.1549.

Schwab, U., et al. (2008). Triacylglycerol fatty acid composition in diet-induced weight loss in subjects with abnormal glucose metabolism-The GENOBIN study. PLOS ONE, 2, e2630. doi: 10.1371/journal.pone.0002630.

Stamler, R., et al. (1978). Weight and blood-pressure findings in hypertension screening of 1-million Americans. JAMA-Journal of the American Medical Association, 240, 1607-1610. doi: 10.1001/jama.240.15.1607.

Tuomilehto, J., et al. (2001). Prevention of type 2 diabetes mellitus by changes in lifestyle among subjects with impaired glucose tolerance. The New England Journal of Medicine, 344, 13431350. doi:10.1056/NEJM200105033441801.

Weckwerth, W. (2003). Metabolomics in systems biology. Annual Review of Plant Biology, 54, 669-689. doi:10.1146/annurev. arplant.54.031902.135014.

Yetukuri, L., et al. (2007). Bioinformatics strategies for lipidomics analysis: Characterization of obesity related hepatic steatosis. BMC Systems Biology, 15(1), 12. 